Instituto Internacional de Investigación y Desarrollo Tecnológico Educativo INDTEC, C.A.

DOI: https://doi.org/10.29394/Scientific.issn.2542-2987.2018.3.7.17.329-352

OAI-PMH: http://www.indteca.com/ojs/index.php/Revista Scientific/oai

\title{
Expresión Oral Emergente en los Estudiantes Durante la Interacción Social
}

Autores: Yuraima Margelis Matos de Rojas Universidad Nacional Experimental Simón Rodríguez, UNESR yuraimatos01@gmail.com Yvo Antonio Barreto Andrade Universidad Nacional Experimental Rafael María Baralt, UNERMB yvobarreto.a@gmail.com María Martina Mejía de Cordero Universidad Nacional Experimental Rafael María Baralt, UNERMB mariamdecordero@gmail.com Gaby Angélica Terán Viera Universidad Nacional Experimental Rafael María Baralt, UNERMB terangaby2012@gmail.com Trujillo, Venezuela

\section{Resumen}

Durante el accionar educativo se dan procesos interactivos entre los estudiantes, permitiendo distinguir expresiones orales, relacionadas con la forma de comunicación interpersonal que se da entre los estudiantes, en la cual utilizan calificativos para identificar y caracterizar a sus compañeros, lo cual puede beneficiar y/o afectarle. De allí que el propósito de la investigación fue: Develar las expresiones orales que emergen entre los estudiantes para una cualificación valorativa en su interacción social. Metodológicamente se abordó desde una visión cualitativa fundamentada en el método etnográfico, con una población de 38 alumnos; desarrollada en cuatro fases: (1) acercamiento a la realidad, (2) recolección de información, a través de la observación y la entrevista; (3) análisis de la información y (4) elaboración del informe final. Del proceso de análisis de la información, se construyeron categorías, tales como: el burlón, el/la craniecito(a), el molestón/fastidioso, el/la solidario(a). Calificativos estos, que según los alumnos eran característicos por su manera de actuar. Entre los valores manifestados se tienen la amistad, el respeto, la sinceridad, el compañerismo. De igual manera, contravalores, tales como; irrespeto, desorden, burla, ofensa, indisciplina, entre otros. Por lo que se recomienda propiciar actividades que le permita al estudiante valorar y respetar a sus compañeros de clase para un convivir en armonía.

Palabras clave: expresión oral; valores sociales; interacción social. 


\title{
Emerging Oral Expression of the Students During Social Interaction
}

\begin{abstract}
During the educational actions are interactive processes between the students, allowing to distinguish oral expressions, related to a form of interpersonal communication that occurs between students, which uses labels to identify and characterize his companions, which may benefit or affect you. From there that the purpose of the research was: unveil oral expressions that emerge among the students for an evaluative qualification in their social interaction. From the point of view of the methodology, it was approached from the qualitative vision based on the ethnographic method, using a population of 38 students; developed in four phases that are: (1) an approach to the reality, (2) collection of information, taking in account observation and interview; (3) analysis of the information and (4) preparation of the final report. From the information related to the analysis process, categories were constructed, such as: the mocker, the craniecito, the annoyance/annoying and the supportive. Such qualifiers, which according to students were characteristic by their way of acting. Among the values were expressed: the friendship, the respect, sincerity, fellowship. Similarly, counter values, such as; disrespect, disorder, ridicule, offense, indiscipline, among others. Therefore it is recommended to promote activities that allow the student to value and respect their classmates for a living in harmony.
\end{abstract}

Keywords: oral expression; social values; social interaction. 


\section{Introducción}

Investigar sobre las acciones que los actores del aula ejecutan cotidianamente, resulta ser interesante y pertinente en los actuales momentos que se intentan cambios en todos los aspectos de la humanidad, especialmente en lo que a educación se refiere; por cuanto permite determinar las debilidades como fortalezas que se generan en el aula de clase. Espacio donde mayormente permanecen docente y alumnos y, en el que diariamente se propician acciones interactivas que llevan a poner de manifiesto valores, al igual que situaciones no muy acertadas durante los encuentros de aprendizaje, que merecen ser investigados y estudiados para darle solución o reforzar los mismos.

De allí, que a diario se oye a los docentes, padres y estudiantes expresarse sobre eventos que se producen en los espacios de aprendizaje, haciendo más énfasis en las dificultades y problemas, que en los beneficios y satisfacciones que puede promover el interactuar social durante el proceso de aprendizaje docente-alumno, donde se llevan a cabo expresiones orales, relacionadas con la comunicación interpersonal que se genera entre los estudiantes y que muchas veces pueden llevar a valorar como desvalorar al otro. Situación a la que no escapan los niños, niñas de la UE "El Cumbe", Municipio Valera, Edo. Trujillo, en donde las docentes indicaron en varias oportunidades que se vienen presentando ciertas dificultades en el interactuar entre los estudiantes lo que a veces no saben cómo gestionar.

Desde esta perspectiva, se hace necesario profundizar en el estudio de los valores que subyacen en los espacios educativos, más cuando Cadenas (2017a, pág. 345), refiere "que los valores en la actualidad, debido a los cambios que se vienen generando han dado lugar a un sistema de valores, cuyo determinante son la competitividad y el individualismo". Aunado a lo señalado por la autora citado, se hace necesario referir que en la Constitución de la República Bolivariana de Venezuela (1999), y en la Ley Orgánica de 
Educación (2009), como realidades legislativas, le designan al sistema educativo venezolano fortalecer los valores de respeto, solidaridad, tolerancia, solidaridad, empatía, entre otros; que como principios rectores de la educación, resguarden la integridad del ser y de interacción en los diferentes espacios donde se desenvuelve el ciudadano venezolano.

Reflexionando sobre lo señalado, surgió como propósito del estudio Develar las expresiones orales que emergen entre los estudiantes para una cualificación valorativa en su interacción social con el fin de sugerir algunas actividades que fortalezcan el proceso interactivo. Estudio que se justifica, considerando que los espacios educativos son privilegiados por el constante interactuar de los actores activos que lo conforman y, donde se educan, construyen y practican valores que ameritan descubrir para contribuir a su mejora. Desde esta perspectiva, el estudio se sustentó en el método etnográfico para visualizar y develar la realidad desde los actores de la investigación. La misma se insertó en la Línea de Investigación Investigadores en Acción Social, IAS, UNESR, Núcleo Valera.

\section{Contexto Teórico}

\subsection{La Interacción social en los espacios de aprendizaje}

Existen diversos investigadores que se han preocupado por el estudio de las interacciones, en el que se destacan los muy conocidos trabajos de Flanders, en los años setenta, dentro de la corriente identificada como análisis de interacción. En la actualidad continúa vigente el interés en investigar no sólo la interacción entre alumno-profesor sino también en el proceso mismo de interacción.

La palabra interacción, es de origen latín y describe un trato entre dos - más personas; ubicándonos en el contexto educativo las personas obviamente serían el docente y los alumnos. Ibáñez (2004), aporta una definición y la conceptualiza, las reacciones adoptadas por las personas hacia 
los otros. Reacción que depende del contexto y de la situación en la que tiene lugar la interacción, así como de la manera como los actores negocien el significado que otorguen al contexto. Definición que está relacionada con la interacción con otros individuos, que lleva a modelar de alguna manera ciertas conductas, de acuerdo con las creencias, costumbres, valores, entre otras. El ser humano está en constante proceso de interacción a lo largo de su vida y, le lleva de acuerdo con Matos (2014), a establecer relaciones entre los diversos actores que hacen vida en cualquier contexto, donde comparten, ideas, conocimientos, experiencias, emociones, de cara a cara o en la distancia por medio de los diferentes medios tecnológicos.

Ubicando la interacción social en el contexto educativo, Guevara (2011a), expresa que es el proceso interactivo que se da entre el estudianteestudiante, estudiante-contenido, y docente-estudiante. De pendiendo la forma en que se genere el proceso educativo es como ocurrirá la interacción. Es decir, es una reciprocidad, verbal o no verbal, que se establece entre los agentes del aula; siendo necesario considerar el contexto, los medios y recursos, el clima, entre otros, como elementos claves y significativos para el proceso de interacción.

La interacción educativa, según Bixio (2001a), es una acción compartida, lo que indica, que las relaciones interactivas docente-estudiante, se deben producir de manera favorable en la medida que entre ellos fluya una comunicación efectiva recíproca. Por esta razón, merece especial atención la consideración de la comunicación como parte de los elementos básicos que permiten entender los procesos de interacción estudiante-profesor, así como estudiante-estudiante.

Para efecto del estudio, se hará énfasis en la interacción estudianteestudiante, sin dejar de considerar la importancia de la interacción docenteestudiante. La interacción estudiante-estudiante, es la que utilizan los participantes o alumnos para ponerse de acuerdo sobre la división de 
funciones y logística de las tareas en equipo. Para Bixio (2001b, pág. 22), así como para Guevara (2011b), este tipo de interacción favorece el trabajo entre pares, a la vez que se produce una influencia educativa importante y enriquecedora de los procesos de construcción de conocimientos. De igual manera, durante el proceso de interacción social entre los estudiantes se propician modos de actuar que los caracteriza y los lleva a manifestar valores y contravalores que pueden beneficiar $\mathrm{y} / \mathrm{o}$ afectar al estudiante.

De allí la necesidad que los docentes como gerentes del aula, promuevan procesos interactivos, que como indica Jáuregui (2017), "generen la mayor cantidad de oportunidades de participación e interacción para los estudiantes, lograr el éxito en el alcance de las metas propuestas". (pág. 141). Proceso en el que se establezcan relaciones interactivas, afectivas y efectivas que beneficien el acto educativo y la formación de ciudadanos con valores y fortalezas para actuar en correspondencia con las exigencias y cambios que se gesten en el país.

\subsection{Los valores sociales}

Los valores sociales, de acuerdo con Bello (2004a), comienzan a formarse en la práctica como un ejercicio individual, los cuales constituyen para cada individuo un compromiso como alguien que actúa en una comunidad de individuos, que desea resultados positivos en su crecimiento personal y su dimensión social. Los valores sociales, según el Ministerio del Poder Popular para la Educación (2007), indica que se forman en una comunidad específica, en cada población y país, como fruto de un proceso de intercambio.

En este proceso de valoración o acto de valorar se hace necesaria la presencia del sujeto como del objeto. Tal como señalan Matos y Pirela (2004a), durante el acto de valoración pueden intervenir aspectos cualificadores que inciden en la disposición del que enseña y el que aprende. Asimismo, en los espacios educativos se vivencian valores sociales durante el 
proceso de aprendizaje, que pueden llevar a los estudiantes a valorar 0 desvalorar acciones y prácticas. Entre ellas tenemos los calificativos que los estudiantes utilizan para referirse a características, comportamientos y actitudes de sus compañeros.

\subsection{Expresión oral}

El habla es la capacidad comunicativa relacionada con la elaboración del discurso oral. La expresión oral para Recasens (2003), constituye una destreza o habilidad de comunicación que no tiene sentido sin la comprensión, sin el procesamiento y la interpretación de lo escuchado. La expresión oral implica la interacción y la bidireccionalidad, en un contexto compartido, y en una situación en la que se deben negociar los significados. La comunicación es un proceso, una acción, basada en unas destrezas expresivas e interpretativas, por lo que la expresión oral debe entenderse como tal, junto a la comprensión oral, la lectura y la escritura.

La expresión oral está relacionada con el acto del habla y a su vez con las formas de comunicación interpersonal que se genera entre los estudiantes, llevándolos muchas veces al uso de calificativos valorativos o atributos que les confiere sobre su modo de actuar en el espacio educativo. De acuerdo con Matos y Pirela (2004b), los calificativos hacen referencia a cualidades, rasgos y propiedades que acompañan al nombre para hacerlo distinto a los demás. Los mismos pueden fortalecer como dañar, es decir, son positivos $y / 0$ negativos. En cuanto a los calificativos, tienen que ver con el acto de valoración, atributos que los estudiantes hacen a sus compañeros, lo cual dependiendo del mismo puede generar burlas y hasta actos violentos que afectan la integridad del estudiante.

De allí que estas expresiones orales pueden llegar a afectar el proceso de interacción que da entre los estudiantes, donde el docente tiene la gran tarea de fomentar la educación en valores propiciando la participación, efectiva 
y coherente, donde se lleve al estudiantado a vivenciar valores que le permita construir desde su acto de valoración de sí mismo y al otro durante el proceso interactivo, dentro y fuera de los espacios educativos. Se trata, como señala Cadenas (2017b), fortalecer valores que le permitan al estudiantado aprender a convivir y compartir con armonía, e interactuar afectivamente en los diferentes espacios en que se desenvuelven.

En este sentido, se trata de fortalecer espacios de participación e integración de los actores que hacen vida en las aulas de clase, donde se fomenten valores, que como señalan Rondón, Peña y Terán (2017), unos valores sociales impregnados desde una desde una postura humanista, que lleve a los ciudadanos actuar corresponsablemente en el proceso de formación de los estudiantes. Que les permita interactuar de forma armónica donde prevalezcan los valores ante que los calificativos que menosprecien a sus compañeros de clase, donde convivan con respeto y solidaridad.

\section{Senda Metodológica}

Ante el objetivo planteado y conociendo las bondades que ofrece el método etnográfico, que según Goetz y Le Compte (1988a), permite describir lo que sucede en grupos intactos, los investigadores abordaron el espacio de aprendizaje de 38 alumnos para adquirir detalles necesarios que le permitieran describir los hechos tales como estaban sucediendo y develar lo que realmente sucedía en el escenario de aprendizaje.

De acuerdo con Goetz y Le Compte (1988b), el método etnográfico, permite la descripción holista de la interacción natural de un grupo en un período de tiempo, que representa fielmente las visiones y significados de los participantes. Asimismo, Martínez (1998a); Rodríguez, Gíl y García (1999a), indican que las aulas por ser escenarios pequeños y en donde acontecen situaciones necesarias de profundizar, favorecen la aplicación de la investigación. 
Ahora bien, la metodología etnográfica permitió develar el proceso interactivo que se generaba entre los actores de las aulas de clase. Metodología, que trae consigo una ardua tarea para registrar los hechos tal y como suceden en la realidad; la misma se desarrolló en cuatro fases: (1) acercamiento a la realidad, (2) recolección de información, a través de la observación y la entrevista; (3) análisis de la información y (4) elaboración del informe final.

La primera fase permitió el acercamiento a la realidad de estudio, solicitando permiso a la docente para realizar la investigación, considerando que en diversas oportunidades la docente expresó los diversos conflictos que a menudo se dan entre los estudiantes. De igual manera, un acercamiento previo con los estudiantes a fin de establecer un primer contacto con los mismos.

Con relación a la segunda fase, que se refiere a la recolección de la información directamente en el campo de trabajo, lo que implicó la utilización de técnicas para la recolección o captura de datos, tales como: la observación, considerada por Martínez (1998b) y Tarrés (2013a), como la técnica clásica primaria y más adecuada por los etnógrafos para adquirir información; observación que fue participativa, por cuanto permitió una mayor interacción y comunicación con los grupos a estudiar, para percibir, comprender y explicar la realidad tal y como se dio. La entrevista, a través de ella se pudo dialogar con los informantes o agentes de estudio, para obtener información de aspectos o situaciones que no habían quedado claros en las observaciones, en especial en los que había necesidad de profundizar.

Los instrumentos que se emplearon para recoger y almacenar la información fueron los diarios de campo, que permitieron hacer anotaciones personales (sensaciones, interpretaciones, reflexiones, etc.) de las observaciones realizadas, así como gestos, actuaciones, entre otras, que se dieron en las aulas de clase. 
La tercera fase 0 análisis de la información, permitió la organización de la información, la transcripción y el análisis de la misma. Taylor y Bogdan (1986), Huberman y Miles (1994), Rodríguez, et al. (1999b), y Tarrés (2013b), consideran el análisis de los datos, como el proceso dinámico y creativo que realiza el investigador por descubrir las relaciones a través de los hechos acumulados. Es decir, reducción de los datos, disponibilidad de los datos y conclusiones/verificaciones.

La triangulación, vista por Calderero y Carrasco (2000), Coffey y Atkinson (2003) y González (2007), como el proceso que permite chequear la información recabada, en la que se hace uso de diferentes métodos, con el fin de contrastar y comparar los hechos, la cual se llevó a efecto durante el proceso análisis de la información, permitiendo comparar y contrastar los hechos registrados en las observaciones y entrevista, a través de un proceso en espiral "ir y venir" (mientras se realizaba la revisión del material), hasta obtener las categorías y subcategorías que identificaron la información recolectada, descubriendo significados, concepciones, sucesos, y en especial, la actuación de cada uno de los actores en el aula.

La cuarta fase o de elaboración del informe final, que permitió la revisión exhaustiva de toda la información para darle coherencia y fluidez a la misma; producto de esta fase, es la difusión de los resultados obtenidos en el estudio.

\section{Resultados}

En cuanto a los resultados obtenidos del análisis de la información obtenida de las observaciones y entrevistas a los estudiantes, se elaboraron categorías en cuanto a los calificativos, así como a los valores presentes en el interactuar entre los estudiantes; las cuales se pueden mencionar:

El burlón, aquel estudiante que estaba pendiente de lo que hacían los demás para decir palabras o expresiones fuertes, que hacían sentir mal a su 
compañero de clase. Entre las situaciones en que el niño burlón expresaba palabras ofensivas a sus compañeros, se pueden citar: "en una actividad que se estaba realizando en la pizarra, uno de los niños se equivoca, cuando se oye decir 'si es gafo no sabe', la maestra parece que no se dio cuenta de la expresión, aunque los demás estudiantes que estaban cerca si lo escucharon", en otro momento "la maestra le pide a una niña que por favor resuelva una operación matemática en la pizarra y ella con su cuerpo tapó los demás ejercicios, a lo que se escucha 'la carne de burro no es transparente', a lo que la maestra le dice ¿qué pasó? y el estudiante responde 'es que no me deja ver', pero por eso no tiene que decir así, el alumno se ríe y sigue escribiendo".

Como se evidencia, son expresiones que el estudiante, según lo que señalan sus compañeros en el minirrelato, mantiene constantemente, por lo que expresaron "a mi amigo lo llamamos el burlón porque todo el tiempo se burla de lo que uno hace"; otro indicó "a Juan lo llamamos el burlón, porque todo el tiempo está pendiente de lo que uno hace para burlarse". Calificativo este que parece no gustarle mucho a Juan, cuando dice "mis amigos se quejan de que yo me burlo de ellos, pero es que no están mosca. Me molesta cuando me dicen ahí va el burlón, yo trato de no decirles cosas, pero es para que se avispen".

Como se puede observar, las situaciones presentadas permiten indicar que al estudiante le molesta el calificativo de burlón, aun sabiendo que él expresa calificativos fuertes a sus compañeros y, que de acuerdo con él es para que se avispen. Calificativo que lleva al irrespeto, siendo éste el contravalor del respeto, el cual, según Rondón (2016a), el docente debe promover el respeto entre los estudiantes y demás actores que hacen vida en la institución; de tal manera, que lleve a respetarse así mismo, a sus compañeros, haciendo los derechos y la dignidad del otro. El docente debe estar cerca del estudiante, conocerlo y así traspasar los linderos se la escuela, es decir, conocer al estudiantado y planificar acciones en función de sus 
debilidades y fortalezas, que lo lleven a orientar y ser modelo de valores que demuestren el respeto por sí mismo y el otro. (Juárez, Moreno, Straka y Lezama, 2003a, pág. 127).

El molestoso o fastidioso; identificado como aquel niño que esperaba que la docente estuviese distraída o saliera del aula para molestar a sus compañeros. Así se refleja en la siguiente situación: "la maestra conversaba con otra docente, y dos alumnos comenzaron a lanzarse papeles y pedazos de goma del borrador, la maestra hace el señalamiento a los dos niños y sigue conversando, una de las gomas de borrar le cae a una niña en la cara y lo acusa, la maestra opta por ponerlo de pie, en forma de castigo". Otra de las situaciones visualizadas fue cuando "la maestra salió del salón y Saúl comienza a lanzar papeles en forma de pelotica, le golpea en la cabeza a otro niño, quien decide ignorarlo y siguió conversando con su compañero cercano; en vista de su actitud, Saúl se levantó y fue hasta donde estaba el niño para golpearlo, llega la maestra y acusan a Saúl, señalando la maestra, ¿viste por qué te castigo?, a lo que Saúl hace gestos de no importarle".

En cuanto a lo señalado en los minirrelatos se visualizaron expresiones, tales como "Saúl es un niño molestoso que fastidia a los demás compañeros", "en el salón existen varios de los compañeros que son molestosos, se aprovechan cuando la maestra sale un momento o cuando llega alguien al salón", "Hay alumnos que molestan mucho, pero la maestra los castiga y ellos pareciera que no les importa". Expresiones que reflejan el desagrado por las molestias que estos estudiantes ocasiones, lo cual los lleva a colocarle el calificativo de molestoso.

Situaciones que reflejan nuevamente, el irrespeto hacia el otro, que en palabras de Saúl de acuerdo con lo ocurrido dice "yo estoy claro en que molesto, pero es porque ellos todo el tiempo me dicen allí va el molestoso, y a veces me echan a mí la culpa de cosas que yo no hago". Expresión que deja entrever la molestia que siente el estudiante ante el calificativo y que más que 
ayudar puede entorpecer la actitud del mismo. Como se refleja en las situaciones señaladas se da el irrespeto al otro, la indisciplina, el desorden, el maltrato; es decir contravalores que de acuerdo con Bello (2008), llevan a transgredir las normas, los deberes y los derechos. En tal sentido, se requiere que el docente promueva valores que contrarresten las situaciones de irrespeto entre los estudiantes

Por otra parte, cabe señalar la actitud de la maestra hacia el estudiante, que de acuerdo con Matos y Pirela (2004c), muchas veces los docentes optan por medidas arbitrarias para mantener el control o la disciplina en el aula. Medida que podría tener doble vertiente, una que lleve al estudiante a tomar conciencia y analizar su comportamiento y la otra que se acostumbre a molestar y opte por hacer caso omiso al castigo.

En ambas situaciones se evidencian contravalores o desvalores que pueden afectar el proceso educativo, lo que es contrario a lo señalado por Latapí (2000), el docente debe fomentar valores que lleven al estudiante a convivir con el otro, a aceptarlo y respetarlo. Igualmente, Rondón (2016b) expresa que los docentes deben fomentar en los estudiantes conductas orientadas a la crítica, al respeto por las otras personas, al compromiso, la solidaridad, etc. Conductas que pueden estar involucradas en una actividad del salón, en la discusión de un tema, en la práctica del deporte, en cualquier actividad que utilice el docente como estrategia para poner en práctica un plan de formación de valores.

El craniecito/inteligente, es visto como aquel niño que más participa en clase y quien obtiene mejores calificaciones. Entre las verbalizaciones que dejan evidencias del mismo, se pueden señalar: "Pedro es el más inteligente, por eso lo llamamos el craniecito", "uno de nuestros compañeros es muy inteligente, cuando la profe hace preguntas difíciles él levanta la mano para intervenir, la maestra siempre lo felicita y, por eso lo llamo el craniecito", "son varios los estudiantes que son inteligentes y por eso les decimos craniecito, a 
veces a ellos no les gusta, porque como dice María 'no me gusta que me llamen así, yo tengo mi nombre' y bueno yo ya no le digo así".

Igualmente, en la entrevista a Pedro llamado "el craniecito", responde de la siguiente manera "mis participaciones son acertadas, todos mis compañeros me miran a mí para que yo participe, eso para mí es fácil, yo lo entiendo". Sin embargo, uno de sus compañeros dice "Pedro se la da de que sabe todo y a veces se equivoca, pero la maestra se la pasa, en cambio María es muy inteligente, ella estudia mucho y nos ayuda cuando no entendemos". Expresiones en las que se refleja la inteligencia, el compartir, la solidaridad, la amistad, el respeto, valores que debe aprovechar la docente para fortalecer la dignidad humana.

Así como estas expresiones, también se recogieron palabras que indicaron el descontento de los calificativos, a lo que una alumna exterioriza "me molesta eso de craniecito, porque hay compañeros que no gozan de los beneficios que le da el ser cráneo o inteligente, muchos de mis compañeros son etiquetados como bruto y eso me molesta".

Ante estas locuciones, se visualizan los calificativos de craniecito, inteligente, pero también de bruto, que como señaló la estudiante los etiquetan, lo cual puede favorecer como desfavorecer, dependiendo la situación. En este sentido, la actitud que el alumno asuma durante las clases es quizás el reflejo del trabajo que realizan, el tipo de interacción, el agrado o desagrado del momento en que se encuentran. Juárez, et al. (2003b, pág. 128). De allí la necesidad de una educación en valores, que lleven al docente a fortalecer valores que ayuden al estudiante a respetar y aceptar al otro sin tener que colocar calificativos que puedan perturbar la conducta del estudiante, en palabras de Juárez, et al. (2003c, pág. 128), "no hay educación sin respeto a las ideas, sentimiento y honra a los demás".

El/la Solidario(a); término que se le asignó a los alumnos que cooperaban y ayudaban a sus compañeros, actitud que se observó en los 
espacios de aprendizaje. Una de las situaciones que se presentó fue "cuando en una actividad que se estaba realizando, uno de los estudiantes se encontraba como distraído y llegó Ana y le dijo ¿qué te pasa?, a lo que el niño le dijo no entiendo, tranquilo yo te explico y se sentó a su lado para explicarle". En otro momento se visualizó cuando, un estudiante en vista de que su compañera no trajo lápiz optó por darle el de él y se levantó a buscar uno prestado". Observaciones que reflejan el compañerismo, la solidaridad y la amistad, valores que fortalecen el interactuar en los espacios de aprendizaje.

De igual manera, en las entrevistas con los alumnos, se recogieron expresiones, tales como: "Ana es muy buena compañera, ella es solidaria, siempre nos ayuda cuando se nos dificulta algo", "hay amigos que le decimos son solidarios, porque cuando a uno le falta algo lo comparten, igual cuando uno no sabe algo nos ayudan o nos avisan de las tareas cuando faltamos". El/la alumno(a) solidario se diferencia en gran medida del alumno burlón, del que molesta; por cuanto el solidario ayuda, coopera con sus demás compañeros.

La solidaridad constituye uno de los valores que el Ministerio del Poder Popular para la Educación (2012) y Bello (2004b), proponen como esencial para practicar en los espacios educativos y el acontecer diario de toda persona. Expresión oral y/o calificativo que puede de alguna manera contribuir a entablar relaciones afectivas entre los estudiantes, haciendo así del proceso interactivo un momento impregnado de valores.

\section{Actividades que se sugieren para fortalecer los valores y evitar calificativos que afecten el interactuar afectivo y efectivo entre los estudiantes.}

Considerando las categorías o resultados obtenidos de la investigación, el/las investigadoras sugieren a las docentes las siguientes actividades: 
- Promover a través de la lectura, la proyección de videos, la práctica de un habla valorativa que fomenten la solidaridad, el respeto, la responsabilidad y la amistad entre los compañeros de clase.

- Propiciar actividades recreativas que lleven a practicar los valores antes señalados en su proceso de comunicación e interacción.

- Escenificar momentos que lleven a reflexionar entre los estudiantes la puesta en práctica de expresiones orales o calificativos que pueden afectar la integridad del estudiante.

- Realizar actividades expositivas, donde los estudiantes a través de la selección de un valor determinado, lo desarrolle de forma creativa.

- Realizar carteleras, afiches, material impreso, que lleve a fomentar la práctica de valores sociales para una comunicación efectiva y un mejor interactuar en la medida que fortalecen las relaciones personales entre el estudiantado.

- Evaluar en conjunto con los estudiantes las actividades desarrolladas y solicitar sus aportes para nuevas actividades a desarrollar.

\section{Reflexiones generales}

Ante las evidencias señaladas, se puede decir que, desde el habla de los estudiantes, emergieron expresiones orales, que posiblemente para ellos es normal al interactuar y referirse al otro por sus cualidades, tales como el burlón, el molestoso o fastidioso, el craniecito/inteligente, el/la solidario (a), los cuales llevan implícitos valores y contravalores. En cuanto a los contravalores: irrespeto, desorden, burla, ofensa, indisciplina, maltrato, entre otros, que pueden afectar, en la mayoría de los casos, el comportamiento de los estudiantes; así mismo, se evidenciaron en el interactuar de los estudiantes valores como: solidaridad, compañerismo, amistad, apoyo, sinceridad, respeto, cooperación, la inteligencia. 
Dada esta situación, se puede decir que la docente desempeña un papel importante en el acto de valoración que realizan los estudiantes durante los encuentros de aprendizaje, pues se evidenció que en diversas ocasiones hizo uso del castigo como medida disciplinaria, lo cual lleva al rechazo por parte del estudiante ante tal situación. Manifestando así un contravalor como es el castigo, maltrato, uso de poder. Sin embargo, hubo momentos donde manifestó comprensión ante situaciones de los estudiantes, el respeto cuando indicaron no entender y ella volvió a explicar el contenido, el apoyo; reforzamiento de valores sociales que los llevó a la práctica, como la amistad, el amor por sí mismo y por el otro.

Desde los resultados obtenidos a través de la etnografía como método de investigación, se puede indicar que más que un método es una herramienta de la cual el docente debe valerse para descubrir y registrar los acontecimientos que se suscitan en los espacios de aprendizaje y, así tratar de comprender las situaciones que se le presenten y poder buscar alternativas de solución. Por lo que es necesario recomendar a la docente, como a todos aquellos docentes que deseen fortalecer los valores, el uso de diversas estrategias y actividades que lo lleven a vivencias valorativas, donde se aprenda a respetar, a aceptar al otro sin tener que utilizar calificativos que pueden afectar el desarrollo integral del estudiante.

De igual manera, que las docentes reflexionen sobre su propia práctica educativa, pues el uso del castigo como un contravalor, puede afectar la actitud del estudiante, por lo que debe ser creadora de estrategias motivadoras, propiciando un ambiente de diálogo, intercambio de ideas, de participación, de igualdad, haciendo el acto educativo interesante y formativo para los estudiantes.

\section{Referencias}

Bello, J. (2008). Valores para construir una ética. Taller para la edificación 
de un mundo posible. Venezuela: Melvin, C.A.

Bello, J. (2004a,b). Valores esenciales para la vida en la familia y en la comunidad. Venezuela: Biblioteca Básica temática.

Bixio, C. (2001a,b). Enseñar a Aprender. Crear un espacio colectivo de enseñanza-aprendizaje. Serie Educación. Santa Fe, Argentina.

Cadenas, Y. (2017a,b). Capacitación en Valores para Fortalecer la Convivencia Social y Ciudadana. Revista Scientific, 2(3), 343-360. Recuperado de:

https://doi.org/10.29394/scientific.issn.2542-2987.2017.2.3.19.343-360

Calderero, J. \& Carrasco, C. (2000). Aprendo a investigar en educación. Madrid: Ediciones RIALPS, S.A.

Coffey, A. \& Atkinson, P. (2003). Encontrar el sentido a los datos cualitativos. Estrategias complementarias de investigación. Medellín, Colombia: Universidad de Antioquia.

Constitución de la República Bolivariana de Venezuela (1999). Gaceta Oficial $\mathbf{N}^{\circ}$ 5.453. Marzo 24, 2000. Caracas, Venezuela.

Goetz, J. \& Le Compte, M. (1988a,b). Etnografía y diseño cualitativo en investigación educativa. Madrid: Morata.

González, F. (2007). Investigación cualitativa y subjetiividad: Los procesos de construcción de la información. México: McGraw-Hill.

Guevara, I. (2011a,b). La interacción en el aprendizaje. Revista Divulgación Científica y Tecnológica de la Universidad Veracruzana. Vol. XXIV, N.은 1. México: La Ciencias y el Hombre. Recuperado de:

https://www.uv.mx/cienciahombre/revistae/vol24num1/articulos/interac cion/

Huberman, A \& Miles, M. (1994). Data Management and Analysis Methods. En Denzin, N. Ei Lincoln (Eds) Hanbook of Qualitative Research. California: Sage Publications.

Ibáñez, T. (2004). Introducción a la Psicología Social. España: UOC. 
Jáuregui, F. (2017). Estrategias Aplicadas por los Docentes como Gerentes de Aula para el Fortalecimiento de los Aprendizajes. Revista Scientific, 2(4), 137-156. Recuperado de: https://doi.org/10.29394/scientific.issn.2542-2987.2017.2.4.8.137-156 Juárez, J., Moreno, A. Straka, T. \& Lezama, J. (2009a,b,c). Educar para vivir.

Cuatro enfoques desde la educación en valores. (2da. Ed) Caracas: Paulinas.

Latapí, P. (2000). Tolerancia: la virtud que nos falta. Revista Proceso, Núm. 1236. México: Ediciones del D.F. Recuperado de:

http://www.proceso.com.mx/284807/tolerancia-la-virtud-que-nos-falta Ley Orgánica de Educación (2009). Gaceta Oficial N. 5.929. Extraordinaria del 15 de agosto de 2009. Caracas, Venezuela.

Martínez, M. (1998a,b). La Investigación Cualitativa Etnográfica en Educación. Venezuela: Texto.

Matos de R., Y., \& Pirela de O., D. (2004a,b,c). ¿Qué valoran los docentes y alumnos en el proceso y enseñanza de la Matemática? Revista de Educación y Ciencias Humanas. Año XII, N. 22, enero-junio. Venezuela: Universidad Nacional Experimental Simón Rodríguez.

Matos de R., Y. (2014). Actividades de participación para una recreación sana sustentable. Revista Sustentabilidad al Día. N.ㄹ 2. enero-junio. Trujillo, Venezuela: Universidad Valle del Momboy.

Ministerio del Poder Popular para la Educación. (2007). Diseño Curricular del Sistema Educativo Bolivariano. Caracas, Venezuela: Autor.

Ministerio del Poder Popular para la Educación (2012). Currículo Básico Nacional. Programa de Estudio. II Etapa de Educación Básica. Venezuela.

Recasens, M. (2003). Comprensión y expresión oral. Barcelona: Editorial Ceac S.A.

Rodríguez, G.; Gil, J., \& García, E. (1999a,b). Metodología de la 
Investigación Cualitativa. Málaga: Ediciones Aljibe.

Rondón, A., Peña, N., \& Terán, G. (2017). Los Valores Sociales desde las Voces de los Actores Socio-Educativos. Revista Scientific, 2(3), 7698. Recuperado de:

https://doi.org/10.29394/scientific.issn.2542-2987.2017.2.3.4.76-98

Rondón, A. (2016a,b). Valores sociales en espacios humanísticos; Un acercamiento socio-educativo. Tesis Doctoral. Mención Publicación. Cabimas, Venezuela: Universidad Experimental Rafael María Baralt.

Tarrés, L. (2013a,b). Observar, escuchar y comprender sobre la tradición cualitativa en la investigación social. México: FLACSO.

Taylor, S. \& Bogdan, R. (1986). Introducción a los Métodos Cualitativos de Investigación. Buenos Aires: Editorial Paidos. 
Nacida en Venezuela. Licenciada en Educación

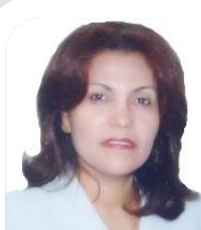
Integral, Mención Matemáticas, Magister en Ciencias de la Educación. Mención Investigación Docencia. Magister en Tecnología y Diseño educativo, Magister en Educación Robinsoniana, Dra. En Ciencias de la Educación. Docente investigador en la Universidad Nacional Experimental Simón Rodríguez, (UNESR) Núcleo Valera, Edo. Trujillo (Jubilada). Participante de la Línea de investigación: Investigadores en acción Social (IAS), Facilitadora en cursos de metodología, Seminario de investigación, Valores, Servicio Comunitario, Proyecto de Investigación, administración de la educación Básica, Ética y valores en la investigación, gerencia educativa, entre otros cursos, en las Universidades: Simón Rodríguez, Valle del Momboy, Rafael María Baralt (Especialidad, Maestría y Doctorado). Jurado evaluador y tutora de Trabajo Especial de Grado y Tesis, Evaluadora de trabajos de ascenso, artículos arbitrados en Revistas nacional e internacional. Actualmente miembro del PEII, NIVEL B. 


\section{Yvo Antonio Barreto Andrade \\ e-mail: yvobarreto.a@gmail.com}

Nacido en Venezuela. Lcdo. En Educación Mención

Filosofía: IUSPO/ UCAB, Especialista en Planificación y

Evaluación: UNIVERSIDAD SANTA MARÍA. Magister en

Gerencia Educacional: UPEL, Cursante del Doctorado

en Educación: Universidad Nacional Experimental

Rafael María Baralt, UNERMB. Actualmente:

Coordinador de Dpto., Orientación y Bienestar Estudiantil Escuela Técnica Agro- Industrial Profesor. Pedro Albarrán. Parroquia el Araguaney, Municipio Andrés Bello. Estado Trujillo- Venezuela. Ponente a nivel nacional. 


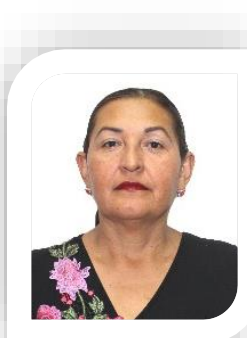

Nacida en Venezuela. Lcda. Educación Integral Mención Lengua. Especialista en Currículo, Diplomado en Supervisión Educativa, Magíster en Gerencia Educativa, Estudiante del Doctorado en Educación en la Universidad Nacional Experimental Rafael María Baralt, UNERMB. Institución donde labora U.E. Los Caños, Municipio Andrés Bello del Estado Trujillo. 
Nacida en Venezuela. Actualmente docente en el Liceo la Línea de Investigación IAS, Universidad Nacional Experimental Simón Rodríguez, Núcleo Valera, Venezuela. 